\title{
Análisis de cinco estudios de caso y su influencia en el ámbito de la Educación Infantil
}

\author{
Estefanía Castillo Ávila - Universidad de Granada \\ Lucía García Soto - Universidad de Granada \\ Noelia Lancha Moriel - Universidad de Granada \\ Carmen Machado Herrera - Universidad de Granada \\ Julia Maldonado Castilla - Universidad de Granada \\ Mirian Hervás Torres - Universidad de Granada
}

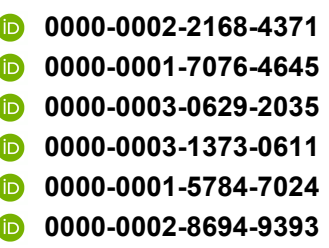

Recepción: 12.12.2018 | Aceptado: 25.01.2019

Correspondencia a través de ORCID: Mirian Hervás

iD 0000-0002-8694-9393

Citar: Castillo, E., Garcia, L, Lancha., Machado, C., Maldonado, J. y Hervas, M. (2019). Análisis de cinco estudios de caso y su influencia en el ámbito de la Educación Infantil. ReiDoCrea, 8, 55-79. []

\begin{abstract}
Resumen: El proceso de investigación constituye uno de los grandes retos para el alumnado universitario, ya que se enfrenta a un trabajo de carácter científico, donde han de poner en práctica todos los conocimientos, competencias, habilidades y destrezas adquiridas a lo largo de su formación académica. Método: Este estudio consiste en la presentación de los resultados obtenidos de cinco Estudios de Casos, los cuales fueron implementados mediante un programa de intervención con alumnado de Educación Infantil que presentaban dificultades en su progreso escolar y/o personal. Resultados: Los resultados indican altos progresos y mejoras en los diferentes estudios de casos una vez finalizaron los programas de intervención, ya que se redujeron conductas no deseadas (p.e., se distrae menos en la realización de las tareas) o en otras ocasiones, aumentaron comportamientos adecuados (p.e., permanece sentado durante la asamblea). Conclusiones: Las aportaciones indican la ardua tarea de ajustar el nivel de desarrollo a las necesidades detectadas, y proponer estrategias adecuadas y efectivas.
\end{abstract}

Palabras clave: Aprendizaje activo | Necesidades educativas

\section{Analysis of five Case Studies and their influence in the field of early Childhood Education}

\begin{abstract}
The research process is one of the greatest challenges for university students, since they are faced with scientific work, where they must put into practice all the knowledge, competencies, abilities and skills acquired throughout their academic training. Method: This study consists of the presentation of the results of five Case Studies, which were implemented through a program of intervention with students of early childhood education who had difficulties in their school and/or personal progress. Results: The results indicate a great progress and improvements in the different case studies once the intervention programs were completed, since unwanted behaviors were reduced (e.g., less distracted during completion of the tasks) or at other times, appropriate behavior increased (e.g., remains seated during assembly). Conclusion: The contributions indicate the arduous task of adjusting the level of development to the needs identified and propose suitable and effective strategies.
\end{abstract}

Keywords: Active learning | Educational needs

\section{Introducción}

Actualmente la sociedad se haya en continua transformación, por ello, la Universidad ha de ajustarse con las dinámicas sociales, culturales, políticas, económicas y educativas propias de cada época, lo cual supone poner un nuevo rumbo en la misión de la Educación Superior hacia la formación de personas competentes, con capacidad de adaptación a los cambios y toma de decisiones que ofrezcan respuestas creativas, óptimas y éticas. Desde esta perspectiva, la reforma impulsada desde el Proceso de Bolonia, y la creación del Espacio Europeo de Educación Superior, han propulsado el trabajo hacia el aprendizaje permanente y la enseñanza por competencias, produciendo cambios a nivel de estructura curricular, organizativa y estructural (Mateo, 
2000) del proceso de enseñanza-aprendizaje centrado en un modelo de aprendizaje por competencias por parte del alumnado. Para comprender este paradigma, se ha de conocer el concepto de competencia, su finalidad e implicación en el ámbito educativo. Inicialmente, se empleó bajo la interpretación de diferentes perspectivas teóricas, que han tratado de darle un significado y determinar cuales son las competencias que debería de adquirir el alumnado universitario, reflexión sobre su definición y relaciones con otros términos similares.

El concepto competencia desde el proyecto de Definición y Selección de Competencias -DeSeCo- de la Organización para la Cooperación y el Desarrollo Económicos (Rychen y Hersh, 2002), representa:

... la capacidad de responder a demandas complejas y llevar a cabo tareas diversas de forma adecuada. Supone una combinación de habilidades prácticas, conocimientos, motivación, valores éticos, actitudes, emociones y otros componentes sociales y de comportamiento que se movilizan conjuntamente para lograr una acción eficaz. (p. 8).

En este sentido, siguiendo a González y Wagenaar (2003) se podría desglosar en aspectos que tendrían que ver con el "conocer y comprender", lo cuales estarían relacionados con el conocimiento teórico de un campo académico, el "saber cómo actuar", referido a la aplicación práctica y operativa del conocimiento a ciertas situaciones y el "saber cómo ser", relacionado a los valores como parte integrante de la forma de percibir a los otros y vivir en un contexto social. Esta idea está ligada al principio de aprender a aprender (Bolívar, 2009), al igual que al de lifelong learning (aprendizaje a lo largo de la vida), caracterizándose por ser multidimensional y estar relacionado directamente con el contexto en que se expresa. Desde esta perspectiva, la labor investigadora dentro de las enseñanzas universitarias, se presenta como un espacio real y un momento donde el alumnado, puede demostrar su preparación como profesional, mediante el desarrollo de un material curricular, que ponga en práctica su creatividad, conocimientos y competencias que se enmarcan en este proceso, así como el impulso de estrategias de aprendizaje entendidas como una serie de procedimientos que un aprendiz emplea de forma consciente, controlada e intencional como instrumento flexible para aprender significativamente y solucionar problemas (Díaz y Hernández, 2002).

En este sentido, se recopilan cinco Estudios de Casos desarrollados en la titulación del Grado de Educación Infantil de la Universidad de Granada, llevados a cabo en su estancia en los centros de prácticas, durante el curso académico 2017-2018. En este periodo, cada alumna diseño, desarrolló e implementó un programa de intervención dirigido al ámbito de la atención temprana, atendiendo a diferentes necesidades $\mathrm{y} / \mathrm{o}$ dificultades previamente detectadas.

\section{Objetivos}

El objetivo fue conocer las implicaciones que supone el desarrollo de estudios de caso y su relación con la mejora en el desarrollo del aprendizaje e investigación del alumnado de Educación Infantil.

Asimismo, los objetivos propuestos para cada caso concreto fueron los siguientes:

\section{Estudio de casos 1:}

1. Mejorar la autonomía del alumnado.

2. Fomentar la atención y concentración del alumnado. 
3. Mejorar la motricidad fina y gruesa del alumnado.

4. Aumentar la concepción numérica del alumnado.

5. Mejorar la comunicación y comprensión lectora.

Estudio de casos 2:

1. Fomentar la lectoescritura, mediante una intervención basada en el juego.

2. Incrementar la capacidad de atención.

Estudio de casos 3:

1. Aumentar la capacidad de memoria, comprensión y atención.

2. Aumentar un lenguaje y una comunicación adecuada a su edad.

3. Mejorar las rutinas y normas para un buen comportamiento en el aula.

Estudio de casos 4:

1. Aumentar la atención.

2. Mejorar la capacidad de concentración.

3. Mejorar las relaciones sociales entre iguales.

4. Mejorar el control de los impulsos

5. Mejorar la autoestima.

Estudio de casos 5:

1. Aumentar el aprendizaje de una nueva lengua.

2. Mejorar las relaciones con sus compañeros.

3. Mejorar la relación con la tutora.

4. Aumentar el vocabulario en castellano.

\section{Métodos}

La intervención utilizada está basada en cinco Estudios de Casos, el cual es entendido como una herramienta de investigación con la que se mide y se verifican los comportamientos involucrados en el fenómeno estudiado (Yin, 1989).

Asimismo, Stake (2007) indica que "un estudio de casos debe abarcar la complejidad de un caso particular, observa la particularidad y de la complejidad de un caso singular, para llegar a comprender su actividad en circunstancias importantes" (p. 11).

Aunque pueda ser difícil estructurar un estudio de casos en diferentes pasos, Montero y León (2002) desarrollaron cinco fases:

1. Selección y exposición del caso.

2. Elaboración de preguntas.

3. Localización de las bases de datos.

4. Análisis e interpretación.

5. Elaboración de un informe final.

En este sentido, Castro (2010) indicaba que los estudios de casos brindan importantes y valiosos resultados e información del tema en el que se quiera profundizar, pudiendo encontrar respuestas en un ámbito y momento determinado, brindando la oportunidad 
de estudiar directamente a un sujeto y ver su evolución a lo largo de un tiempo concreto.

\section{Participantes}

Los participantes de este trabajo fueron un total 5 alumnos de Educación Infantil de diferentes centros educativos. A continuación, se desarrollan cada uno de ellos.

\section{Estudio de casos 1}

Chico de 6 años que se encuentra aún escolarizado en $3^{\circ}$ de infantil (clase de 5 años), el cual ha repetido una vez y tiene una adaptación curricular de $2^{\circ}$ de infantil. En concreto, presenta una discapacidad intelectual, destacando un retraso madurativo y retraso simple del lenguaje, con leve afectación de la comunicaciónrelación. Se caracteriza por los siguientes aspectos, entre otros:

- Repite lo que dicen los demás.

- Se distrae fácilmente.

- Presenta dificultades al articular grupos consonánticos (p.e., "PL")

- Conoce la recta numérica del 1 al 10 por memorización.

- Tiene movimientos óculo-manuales lentos.

- Le cuesta retener conocimientos.

- Le cuesta iniciar la comunicación con el resto del grupo-clase.

Actualmente, en el centro recibe apoyo tanto del Logopeda (dos veces a la semana) como del profesorado de Pedagogía Terapéutica (tres veces a la semana). Cada sesión tiene una duración, aproximadamente de unos 45 minutos. Tres veces en semana, por la tarde, va a terapia con una psicóloga, aunque se desconoce si es un centro privado o público.

En cuanto a sus antecedentes familiares, cabe decir que tiene dos hermanas. Una de 12 años con trastorno del desarrollo y Trastorno de Déficit de Atención con Hiperactividad (TDA/H) inatento y otra de 14 años que tuvo un seguimiento. Además, hay que destacar que su padre presenta dificultades en el aprendizaje.

\section{Estudio de casos 2}

Alumna de 5 años que se encuentra escolarizada en un $3^{\circ}$ de Infantil (clase de 5 años) en la que se ha observado dificultades en el ámbito de la lectoescritura y la atención. Se caracteriza por los siguientes aspectos especialmente:

- Inatención.

- Se distrae con el material de clase.

- Juguetea con sus manos.

- Se tira al suelo.

- Molesta a resto de la clase.

Esta alumna proviene de una familia con un nivel socio-económico medio, y se encuentra en una fase de superación por la separación de sus padres. Asimismo, semanas antes de acabar la investigación se detectaron nuevas conductas: movimientos involuntarios que causaban tensión muscular, o tener la mirada perdida. 
Estudio de casos 3

Niño de 3 años y medio que presenta retraso madurativo. Su diagnóstico fue realizado por su pediatra. Las dificultades observadas son:

- Limitaciones en el aprendizaje, lo cual le está provocando que no logre llevar el mismo ritmo de progreso que sus compañeros.

- Presenta problemas a la hora de expresarse.

- No vocaliza correctamente.

- Posee un vocabulario reducido.

- Muestra problemas de atención y comprensión sobre todo para realizar las diferentes tareas y actividades.

Actualmente cuenta con la ayuda de una logopeda y profesora de apoyo, las cuales trabajan con bastante frecuencia en las aulas específicas con él. En otras ocasiones acude una docente al aula para observar su desarrollo con los iguales y mostrar apoyo a la docente-tutora.

\section{Estudio de casos 4}

Niño de 4 años que presenta síntomas de TDA/H, aunque no está diagnosticado por ninguna entidad competente. Entre sus comportamientos se encuentran las siguientes:

- Incapacidad de prestar atención en las tareas a realizar.

- Imposibilidad de acabar las tareas.

- Dentro del aula se relaciona casi con todos sus compañeros sin problemas, exceptuando con algunos compañeros con los que apenas habla.

- En los recreos siempre juega junto a su prima (un año menor que él) y cuando la maestra no lo vigila, aprovecha para salir de clase y reunirse con su prima.

Por otro lado, destacar la situación de desestructuración de la familia, la llegada de un nuevo miembro, el poco tiempo que pasa con sus padres y el hecho de que suele dormir con su abuela (adulto con el que mayoritariamente permanece).

\section{Estudio de casos 5}

Alumna, de 4 años de edad, proviene de California (Estados Unidos) y lleva en el país de acogida (España) un año. Emigró a este país junto a sus padres cuando tenía 3 años, y comenzó a ir al centro educativo en el tercer trimestre del primer curso del segundo ciclo de Educación Infantil. Entre las dificultades encontradas se destacan:

- Capacidad limitada para comunicarse en la lengua española con soltura.

- La comprensión del idioma es adecuada (comprensión y realización de órdenes sin dificultad), pero incapacidad de establecer una comunicación con otro igual.

- Articulación de algunas palabras en castellano (básicas y mezcladas con su lengua materna, esto es, en inglés). 
- Dificultad de responder a preguntas de respuestas complejas, realizando sólo frases sencillas y con respuesta de monosílabos "sí" y "no".

- La relación con la tutora y grupo de iguales no es adecuada al no entender y poder expresarse en español, llegando al aislamiento.

- Cruza miradas con sus compañeros de mesa y se ríe sólo cuando la ocasión lo requiere, sin articular palabra ni mantener contacto visual.

Además, evita acercarse y hablar con su tutora, ya que cuando lo hace, esta mantiene una actitud diferente comparada con el resto de sus compañeros.

\section{Instrumentos}

El instrumento utilizado para para cada caso de esta investigación fue una hoja de Registro de Observación. Este instrumento fue elaborado ad hoc, cuya finalidad fue registrar todas aquellas dificultades que observaron, para posteriormente categorizar aquellas conductas o dificultades que son necesarias mejorar.

- El estudio 1 se compuso de un total de 46 ítems, divididos en cuatro áreas del desarrollo: (a) área cognitiva: 12 ítems, (b) área lógico-matemática: 10 ítems, (c) área de psicomotricidad: 10 ítems, y (d) área lingüística: 14 ítems. El grado de conducta, oscilaba entre 1 y 4 ( $1=$ nada, $2=$ regular, $3=$ bien y $4=$ muy bien $)$ (Anexo 1).

- El estudio 2 se organizó de un total de 35 ítems, divididos en cuatro categorías: (a) Lectura: 11 ítems; (b) Escritura: 8 ítems; (c) Atención: 9 ítems; y (d) Comportamiento: 7 ítems. El grado de conducta, oscilaba entre 1 y 4 ( $1=$ nada, $2=$ a veces, $3=$ a menudo y $4=$ siempre) (Anexo 2 ).

- El estudio 3 tenía un total de 36 ítems dividido en 2 áreas: (a) lenguaje y la comunicación: 17 ítems, y (b) funciones cognitivas: 19 ítems. El grado de conducta, oscilaba entre 1 y 4 ( $1=$ nunca, $2=$ frecuentemente, $3=$ a veces y $4=$ siempre) (Anexo 3).

- El registro del estudio 4 constó de 18 ítems en total, divididos en 5 bloques de contenidos: (a) atención: 3 ítems, (b) concentración: 2 ítems, (c) impulsividad: 6 ítems, (d) relaciones sociales: 6 ítems, y (e) autoestima: 1 ítem. El grado de conducta, oscilaba entre 1 y 4 ( $1=$ nunca, 2 = alguna vez, $3=$ casi siempre y $4=$ siempre) (Anexo 4).

- El estudio 5 constaba de ocho ítems, los cuales se dividen en dos categorías: (a) idioma: 4 ítems, y (b) socialización: 4 ítems. El grado de conducta, oscilaba entre 1 y $5(1=$ nunca, $2=$ pocas veces, $3=$ con frecuencia, $4=$ casi siempre y $5=$ siempre) (Anexo 5).

\section{Procedimiento}

El desarrollo de esta intervención (en los 5 casos) se elaboró en 4 fases diferenciadas: fase de observación y análisis de necesidades, fase de diseño de la intervención, fase de implementación y fase de recogida de datos y evaluación.

Durante la primera fase, realizada desde el 1 de febrero de 2018 hasta el día 15 de febrero de 2018, donde se observaron aquellas conductas, necesidades y/o dificultades de los sujetos, pasando a su análisis. Mediante un proceso inductivo que partió de un registro narrativo, entendido como una descripción de bajo nivel realizada a través del lenguaje oral o escrito que es propia de las fases iniciales de la observación, y que se caracteriza por su falta de estructura (Berrocal, 2016, p. 5), se registraron todas las características relevantes que presentaba cada sujeto en cada 
estudio, proporcionando información sobre aquellas áreas que deberían trabajarse, y aportaba información para elaborar posteriormente un registro semisistematizado y diseño de las acciones.

En la segunda fase, diseño de la intervención, se llevó a cabo desde el 13 de febrero de 2018 hasta el 28 de febrero de 2018, realizándose dos actuaciones: (a) diseño del registro de observación, y (2) diseño de las actividades. El registro de observación fue utilizado como una herramienta de seguimiento temporal con el conocer la evolución de cada caso, y estudiado en cuatro momentos diferentes -(1) febrero antes de iniciar las sesiones, (2) marzo, (3) abril y (4) mayo una vez finalizadas las intervenciones-. Paralelamente, se elaboraron las actividades propias de la intervención, las cuales estaban centradas en las necesidades detectadas, esto es, para el estudio 1 el área lingüística, la lógico-matemática, psicomotricidad, y cognitiva (especialmente en atención y concentración), el estudio 2 la lectura-escritura, atención y comportamiento, el estudio 3 el lenguaje, la comunicación y las funciones cognitivas, el estudio 4 la concentración, relajación, socialización, autoestima y especialmente las habilidades sociales (respeto e impulsividad), y finalmente para el estudio 5, la mejora del idioma español y socialización.

Asimismo, en esta fase se optó por una metodología individualizada mayoritariamente, con la finalidad de alcanzar los objetivos programados para cada caso.

En la tercera fase, se procedió a la intervención propiamente dicha a través de las actividades diseñadas en la fase anterior. Este momento comprende el periodo del 1 de marzo de 2018 hasta el 9 de mayo de 2018. Las actividades fueron adaptadas a las necesidades detectadas y se tuvo en cuenta las posibles dificultades que podrían surgir durante su desarrollo.

Durante esta fase, en el estudio 1 se realizaron 14 sesiones (12 individuales y 2 grupales), asimismo debido al horario escolar del alumnado, sólo se pudo trabajar dos días a la semana (lunes y miércoles después del recreo), con una duración entre treinta y sesenta minutos aproximadamente, y así no interferir con las sesiones con el logopeda. En el estudio 2 se realizaron un total de 51 actividades (49 individuales y 2 grupales), realizándose semanalmente de lunes a jueves, con una duración de treinta minutos aproximadamente, no obstante, aunque se planificó y propuso un horario concreto de trabajo, estas no pudieron realizarse siempre a la misma hora, debido a incompatibilidad con la programación de la tutora del aula, siendo realizadas algunas después de la asamblea y otras después del recreo. En el estudio 3 se realizaron 50 actividades (36 individuales y 14 grupales), ejecutándose de lunes a viernes con una duración aproximada de media hora. En el estudio 4 se realizaron un total de 49 actividades (28 individuales y 21 grupales), comprendiendo entre los treinta y sesenta minutos, y llevadas a cabo entre dos y tres días por semana, dependieron del tiempo disponible en el aula para no interferir con la programación del aula. En el estudio 5 se realizaron 27 actividades (9 individuales y 18 grupales), realizándose durante tres días a la semana, con una duración aproximada de unos cuarenta minutos.

En la cuarta y última fase, se recogieron todos los resultados obtenidos con los que evaluar el desarrollo de la intervención, es decir, conocer los cambios que se han producido, avances, mejoras y dificultades encontradas, además de conocer si la intervención ha tenido el efecto esperado o por el contrario serían recomendable para futuras intervenciones realizar diferentes adaptaciones. 


\section{Análisis de datos}

El análisis utilizado para este estudio ha sido de carácter cualitativo y cuantitativo, mediante los cuales se han recabado la información de las diferentes observaciones realizadas a los sujetos.

El análisis cualitativo se propone cuando se quieren observar los comportamientos que un sujeto ha realizado ante determinadas actividades y como indica Strauss y Corbin (2002), un análisis cualitativo es un proceso no matemático de interpretación, en el cual se interpretan acciones, lenguaje, hechos funcionalmente relevantes y finalmente los sitúa en una correlación con el más amplio contexto social (Martínez, 2011)

Por tanto, a través de estos análisis se conocerán conceptos y relaciones en los datos y posteriormente serán estudiados.

\section{Resultados}

Los resultados registrados finalizadas las intervenciones fueron los siguientes:

\section{Estudio de casos 1}

Los resultados obtenidos comparando el primer registro -mes de febrero- con el segundo -mes de marzo- se observan mejorías. En el caso del área lingüística se aprecia la distinción de las vocales y consonantes, es más participativo en la asamblea y mantiene un número mayor de diálogos en clase. Respecto al área lógico-matemática, ha adquirido los conceptos de largo, corto, mucho y poco, además los conceptos de grande, mediano y pequeño los domina a la perfección, mientras que la recta numérica, la conoce de manera memorística debido a que el concepto de cantidad aún no lo ha adquirido. Mientras, en el área de psicomotricidad, se observó una leve mejora con respecto al control postural, la adquisición del concepto de lateralidad, lo cual ha revertido en que sus movimientos óculo-manuales fueran más fluidos. Finalmente, en el área cognitiva, los resultados indican una mayor concentración, y ser capaz de identificar sentimientos, tanto en sí mismo como en sus compañeros.

Asimismo, los resultados registrados entre el segundo -mes de marzo- y el tercer registro -mes de abril- también reflejan mejoras en las diferentes áreas observadas. En el área lingüística, mejoró su comunicación. En el área lógicamatemática, se observó la adquisición completa de los conceptos de largo y corto y mejorías en los conceptos de día y noche. Respecto al área de psicomotricidad, adquirió un mayor conocimiento sobre los elementos de su cuerpo y la lateralidad del lado izquierdo. Mientras que, en el área cognitiva, mejoró en aspecto como la retención de algunos conocimientos de las lecturas comprensivas realizadas, se distrae menos y aumentó su capacidad de concentración.

Los resultados obtenidos en entre el tercer -mes de abril- y cuarto registro -mes de mayo-, también indican mejorías, pero en menor grado. En el área lingüística, el alumnado consiguió memorizar rimas cortas y distinguir algunos fonemas. Sin embargo, en el área de la lógico-matemática, se apreciaron retrocesos relacionados con el contenido de la recta numérica. En el área de psicomotricidad, los resultados indican un control postural inadecuado, incluso sus movimientos óculo-manuales no son tan fluidos como en ocasiones anteriores. Por último, en el área cognitiva, se registró la imitación de acciones 
ante algunas tareas y también se apreciaron mayores distracciones en la realización de las tareas. Finalmente, y a pesar, de los inconvenientes surgidos durante el último mes, comparado con el primer mes, se registró un aumento de resultados positivos (Figura 1 ).

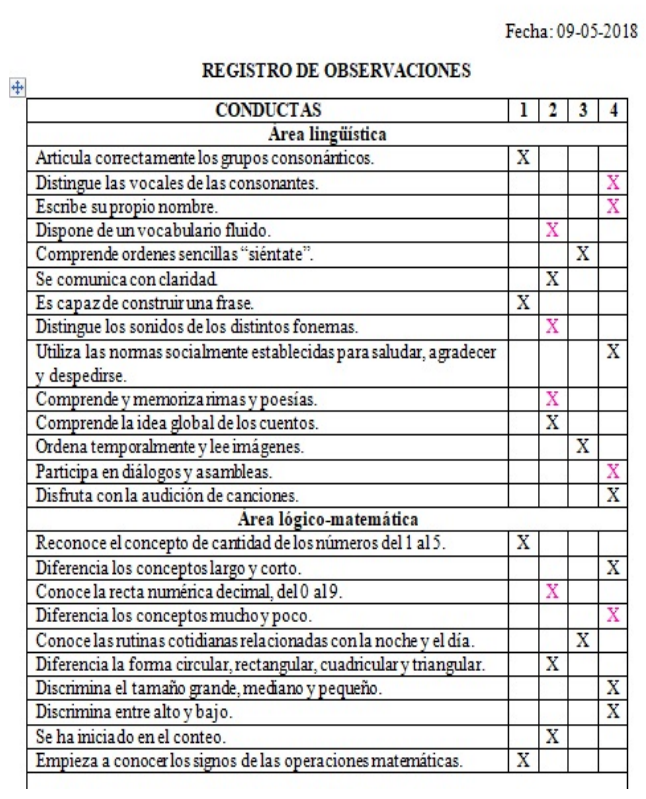

\begin{tabular}{|c|c|c|c|c|}
\hline \multirow{2}{*}{\multicolumn{5}{|c|}{ Área de psicomotricidad }} \\
\hline & & & & \\
\hline Tiene adquirida la concepciónde su propio cuerpo. & $\mathrm{X}$ & & & \\
\hline Tiene fluidez en los movimientos óculo-marnules. & & $\mathrm{X}$ & & \\
\hline Tiene un buen trazo. & & & $\mathrm{x}$ & \\
\hline Es capazde moverse con facilidadpor el espacio. & & $\mathrm{x}$ & & \\
\hline Identifica ellado derecho de su cuerpo. & & $\mathrm{X}$ & & \\
\hline Identifica ella do izquierdo de su cuerpo. & & $\mathrm{X}$ & & \\
\hline $\begin{array}{l}\text { Conoce algunos elementos extemos e intemos de su cuerpo: } \\
\text { extremidades y corazón }\end{array}$ & & & $\mathrm{x}$ & \\
\hline $\begin{array}{l}\text { Participa activamente en las a ctividades fisicas realizadas al aire } \\
\text { libre. }\end{array}$ & & & & \\
\hline $\begin{array}{l}\text { Controla la direccionalidad en las gra fias de los cinco primeros } \\
\text { cardinales. }\end{array}$ & & & $\mathrm{X}$ & \\
\hline \multicolumn{5}{|l|}{ Àrea cognitiva (atención y concentración) } \\
\hline Retiene conocimientos de un dia a otro. & & $\mathrm{X}$ & & \\
\hline Se distrae con facilidad & & $\mathrm{X}$ & & \\
\hline Comprende las explicaciones. & & & $\mathrm{x}$ & \\
\hline Copia las acciones de sus compañeros. & $\mathrm{X}$ & & & \\
\hline Se relaciona conlos demás. & & $\mathrm{X}$ & & \\
\hline Identifica sentimientos y emociones en sus comp añeros. & & & $\mathrm{X}$ & \\
\hline Identifica sentimientos y emociones en sí mismo. & & & $\mathrm{x}$ & \\
\hline Mantiene la atencón enla tarea. & & 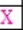 & & \\
\hline Tiene interés por aprender. & & & $\mathrm{x}$ & \\
\hline Evita realizar tareas. & & $\mathrm{X}$ & & \\
\hline Realiza las tareas solo. & & & $\mathrm{x}$ & \\
\hline Trabaja en grupo mostrando a ctitudes de respeto. & & & & \\
\hline
\end{tabular}

Figura 1: Comparación de registros observados meses Febrero vs Mayo

\section{Estudio de casos 2}

Los resultados obtenidos comparando el primer -mes de febrero- con el segundo registro -mes de marzo- indican, en a la categoría de lectura, un incremento de las puntuaciones, resaltando que la pronunciación de las consonantes de forma correcta descendió ligeramente en el mes de marzo. Mientras, en la categoría de escritura, mejoró notablemente, destacando que realiza las vocales sin ayuda, escribe los números bien y copia las frases que se le indica en los deberes. Respecto a la categoría de atención, se observó una disminución leve, encontrando mejoría en el interés de repetir la actividad y presta atención a lo que está haciendo. Por último, en la categoría de comportamiento, se ha mantuvo estable en ambos meses, destacando mejoras en comportamientos como tirarse al suelo y se comporta de forma adecuada en la fila. Los resultados hallados entre el segundo -mes de marzo- y el tercer registro -mes de abril-, indicaron mejoras. En la categoría de lectura, se observó un gran avance en la identificación y pronunciación de vocales y consonantes, además de relacionar la imagen con la palabra que corresponde, dando lugar a un incremento en la puntuación total obtenida. En la categoría de escritura, los resultados indican una leve mejora con respecto al mes de marzo, resaltando un incremento en la escritura de las consonantes adecuadamente. Por otro lado, en la categoría de atención, disminuyó la puntuación, destacando que la conducta juega con sus manos aumentó de manera negativa, mientras que mejoró la atención en las actividades que realiza y no moverse tanto de su asiento. Por último, en la categoría de comportamiento, destacó la mejora de aspectos como respetar el turno de palabra y escucha atentamente a los demás. 
En la comparación entre el tercer -mes de abril- y el cuarto registro -mes de mayo- los datos obtenidos indicaron una gran mejoría en la categoría de la lectura, los cuales amentaron respecto a decir la sílaba que se le indicaba y le daba significado a lo que leía, asimismo disminuyó el uso de ayudas visuales para el reconocimiento de palabras. En la categoría de escritura, se consiguió la máxima puntuación, siendo constantes las puntaciones en el mes anterior y aumentado la escritura de consonantes correctamente. Por su parte, la categoría de atención también mejoró, aumentado los comportamientos de hablar con otros compañeros y pedir volver a hacer la actividad, y disminuyendo comportamientos como sacar la lengua cuando está pensando o querer terminar la actividad. Por último, en la categoría de comportamiento, sólo aumentó la conducta de guardar silencio.

Finalmente, al comparar los resultados del primer con el último registro (meses de febrero y mayo) (Figura 2) se observó que la categoría de lectura incrementó su puntuación considerablemente mejorando sus conductas. Respecto a la categoría de escritura, se incrementaron todas las puntuaciones. En atención, se observó una disminución en las conductas no deseadas, mejorando considerablemente su actitud. Y, en la categoría de comportamiento se observó una leve mejora, manteniéndose estable el comportamiento de sentarse adecuadamente en la hora de la asamblea.

\begin{tabular}{|c|c|c|c|c|c|c|c|c|}
\hline \multirow[b]{2}{*}{ Lectura } & \multicolumn{4}{|c|}{ Febrero } & \multicolumn{4}{|c|}{ Mayo } \\
\hline & 1 & 2 & 3 & 4 & 1 & 2 & 3 & 4 \\
\hline Reconoce las vocales sin ayuda & & $\mathbf{x}$ & & & & $\mathrm{x}$ & & \\
\hline $\begin{array}{l}\text { Identifica las consonantes por si sola cuando se le } \\
\text { pregunta. }\end{array}$ & $\mathbf{x}$ & & & & 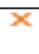 & & & \\
\hline Emite el sonido de las vocales & & & $\mathbf{x}$ & & & & $\mathrm{x}$ & \\
\hline Pronuncia las consonantes de forma correcta & & & $\mathbf{x}$ & & & & $\mathrm{x}$ & \\
\hline Dicen las silabas que se le indican & $\mathbf{x}$ & & & & & & $\mathrm{x}$ & \\
\hline $\begin{array}{l}\text { Usa ayudas visuales para el reconocimiento de } \\
\text { palabras }\end{array}$ & & & & $\mathbf{x}$ & & & $\mathrm{x}$ & \\
\hline $\begin{array}{l}\text { Sabe hacer los movimientos con la boca para } \\
\text { pronunciar una letra }\end{array}$ & & & & $\mathbf{x}$ & & & $\mathbf{x}$ & \\
\hline $\begin{array}{l}\text { Relaciona la imagen que ve con la vocal por la } \\
\text { que empieza }\end{array}$ & & & & $\mathbf{x}$ & & & $\mathrm{x}$ & \\
\hline $\begin{array}{l}\text { Enlaza la imagen con la palabra a la que se } \\
\text { corresponde }\end{array}$ & & & & $\mathbf{x}$ & & & $\mathrm{x}$ & \\
\hline Realiza las actividades sin ayuda & $\mathbf{x}$ & & & & & & $\mathrm{x}$ & \\
\hline Le da un significado a lo que lee & $\mathrm{x}$ & & & & & & & $\mathrm{x}$ \\
\hline Escritura & & & & & & & & \\
\hline Coge el lápiz de manera adecuada & & & $\mathbf{x}$ & & & & $\mathrm{x}$ & \\
\hline Realiza las vocales sin ayuda & & $\mathrm{x}$ & & & & $\mathrm{x}$ & & \\
\hline Escribe las consonantes adecuadamente & & $\mathbf{x}$ & & & & & & $\mathrm{x}$ \\
\hline Repasa los trazos correctamente & & & $\mathbf{x}$ & & & & $\mathrm{x}$ & \\
\hline Escribe los números bien & & $\mathbf{x}$ & & & & $\mathrm{x}$ & & \\
\hline Escribe su nombre & & & & $\mathbf{x}$ & & & & $\mathrm{x}$ \\
\hline Sabe copiar la fecha de la pizarra & & $\mathbf{x}$ & & & & $\mathrm{x}$ & & \\
\hline Copia la frase que se le indica en los deberes & & $\mathbf{x}$ & & & & $\mathrm{x}$ & & \\
\hline Atención & & & & & & & & \\
\hline Se distrae fäcilmente & & & & $\mathbf{x}$ & & & & $\mathrm{x}$ \\
\hline Juega con sus manos & & & & $\mathbf{x}$ & & & & $\mathrm{x}$ \\
\hline Habla con otros compañeros & & & & $\mathrm{x}$ & & & $\mathrm{x}$ & \\
\hline Saca la lengua cuando está pensando & & & & $\mathrm{x}$ & $\mathrm{x}$ & & & \\
\hline Se mueve en la silla & & & & $\mathbf{x}$ & & & & $\mathrm{x}$ \\
\hline Pide volver a hacer la actividad & & $\mathbf{x}$ & & & & & & $\mathrm{x}$ \\
\hline Quiere terminar la actividad & & & $\mathbf{x}$ & & $\mathrm{x}$ & & & \\
\hline Pone atención en 10 que hace & & $\mathbf{x}$ & & & & $\mathrm{x}$ & & \\
\hline Se entretiene con los dibujos de las actividades & & & & $\mathbf{x}$ & & & & $\mathrm{x}$ \\
\hline Comportamiento & & & & & & & & \\
\hline Se tira al suelo & & $\mathbf{x}$ & & & & $\mathrm{x}$ & & \\
\hline Molesta a los compañenos & & & & $\mathbf{x}$ & & & & $\mathrm{x}$ \\
\hline Guarda silencio & & $\mathrm{x}$ & & & & & & $\mathrm{x}$ \\
\hline Se comporta de forma adecuada en la fila & & & $\mathbf{x}$ & & & & $\mathrm{x}$ & \\
\hline Se sienta bien en la Asamblea & & & & $\mathbf{x}$ & & & & $\mathrm{x}$ \\
\hline Respeta los turnos de palabra & & & $\mathbf{x}$ & & & & $\mathrm{x}$ & \\
\hline Escucha atentamente a los demás compañeros & & & $\mathbf{x}$ & & & & $\mathrm{x}$ & \\
\hline
\end{tabular}

Figura 2: Comparación de registros observados meses Febrero vs Mayo

Añadir que en antes de finalizar la intervención se observó una nueva conducta (movimientos acompañados de tensión corporal y manual, tensión en la boca que surgió a raíz de la desaparición de la conducta de jugar con sus manos. Estas conductas, fueron observadas para iniciar una intervención ajustada, ya que se desconocía si tales movimientos eran involuntarios o voluntarios. 
La experiencia los resultados registrados, en el área de lenguaje y comunicación y funciones cognitivas, indicaron progresos. Al comparar el primer (mes de febrero) con el segundo registro (mes de marzo) se observó un progreso en entender las cosas fácilmente, participa en las conversaciones de la asamblea y mantiene el contacto ocular, destacando que comenzó a prestar atención cuando se le hablaba. Respecto a la categoría de funciones cognitivas comparando los meses de febrero y marzo, se observó un progreso en aprender los temas trabajados, se esforzó en hacer las cosas, y mantuvo la atención en la tarea, mientras que el resto de conductas no variaron. Las comparaciones del segundo (mes de marzo) con el tercer registro (mes de abril) se observó un progreso respecto a saber hacer preguntas, expresarse mediante la señalización, presta atención cuando se le habla y el cambio más notable halló en el inicio de una conversación. Respecto al área de funciones cognitivas mejoró en comportamientos como recoger sus pertenencias, y se observó como se esfuerzó en hacer las cosas, o conciencia del castigo. Especialmente destaca la conducta entiende que puede y no puede hacer, la cual pasó de no hacerse a realizarle constantemente.

Finalmente, entre los registros tercero y cuarto (meses de abril y mayo respectivamente) se observó un ligero progreso en la construcción de frases, empezó a pronunciar correctamente e iniciaba conversaciones. Por otro lado, hubo conductas que no produjeron ningún cambio durante la intervención realizada (p.e., uso adecuado de pronombres y formas de expresión normales a su edad). Respecto al área de funciones cognitivas, existió un progreso bastante alto con respecto a los registros anteriores en la conducta entender las repercusiones de sus actos. Asimismo, el cambio más profundo, aunque fue leve, se observó en el esfuerzo en hacer las cosas, controlar el llanto o mantener la atención en la tarea, conductas que previamente no realizaba. A nivel general, los resultados obtenidos indican un progreso continuo, especialmente en las conductas de prestar atención cuando le hablas, ya que al inicio no la realizaba, o el incremento en el esfuerzo por hacer las cosas (Figura 3).

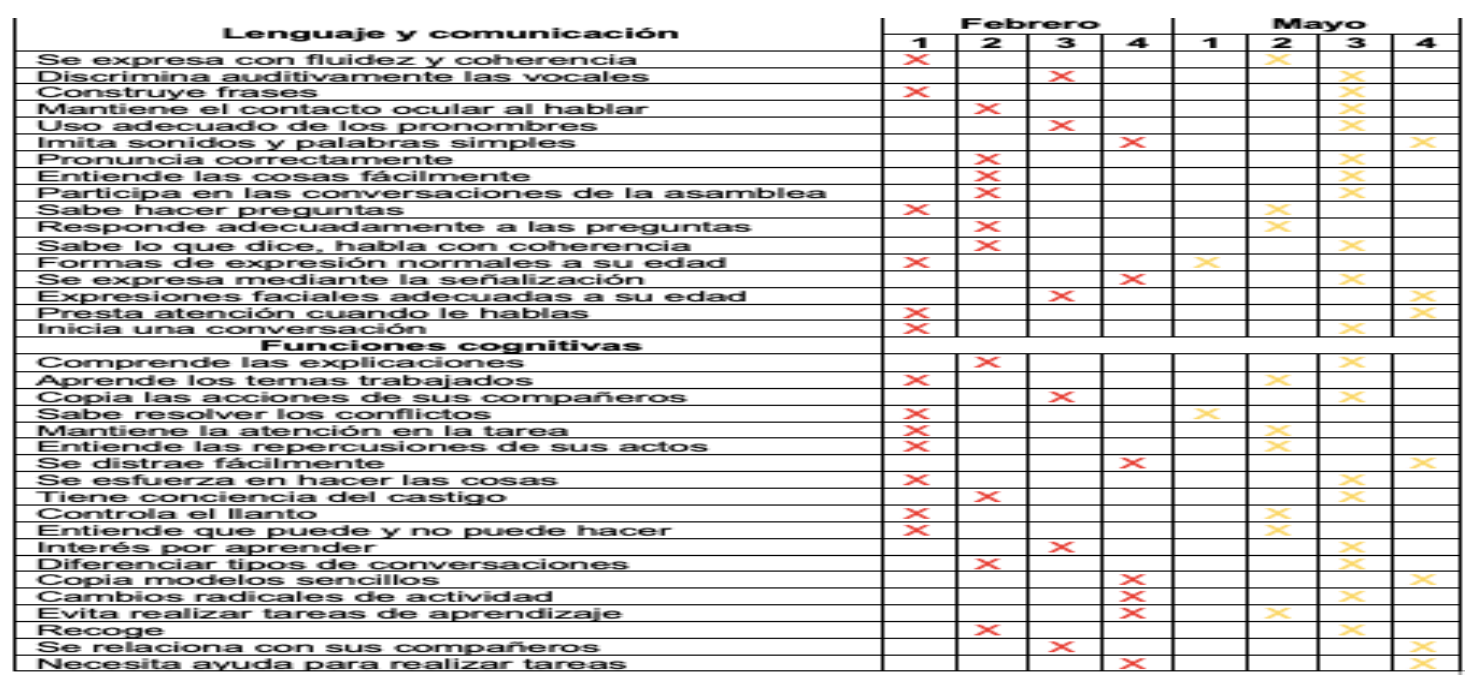

Figura 3: Comparación de registros observados meses Febrero vs Mayo

No obstante, se han hallado comportamientos que no se modificaron durante la intervención, como fue el caso de realizar formas de expresión normales a su edad o saber resolver los conflictos. 


\section{Estudio de casos 4}

Los resultados obtenidos una vez ha finalizado el programa de intervención se observó que, comparando el primer -mes de febrero- con el segundo registro mes de marzo-, se produjeron cambios importantes en los bloques de contenidos de atención en conductas como la mejora en la realización de la tarea sin distracciones, pasando de no realizarlas nunca a hacerlo algunas veces, o del área de impulsividad, como levantarse menos en los momentos que debe prestar atención. Asimismo, en el área de autoestima, la conducta de decirse a si mismo que sus ejercicios están mal descendió levemente.

Respecto a los cambios que se produjeron comparando el segundo -mes de marzo- con el tercero registro -mes de abril- se resumen en variaciones en el bloque de atención, en concreto, en conductas como mirar a la profesora mientras explica la tarea. Mientras, en el bloque de concentración cambió comportamientos como realizar la tarea él solo casi siempre. Y, en bloque de las relaciones sociales, también se observó mejoras con respecto a que sus compañeros se enfadan menos con él.

Sobre la evolución del tercer -mes de abril- con el cuarto registro -mes de mayo- se observó diferencias significativas en cuanto al bloque de atención en comportamientos como escuchar a la maestra atentamente mientras explica la tarea, que siguió mejorando. También se registró progresos en el bloque de impulsividad, reflejado en conductas que descendieron como era pegar a sus compañeros sin ningún motivo. Al igual que en el área de relaciones sociales donde se reflejaron progresos en la conducta de mostrar afecto por sus compañeros.

Por último, al comparar el registro inicial (mes de febrero) con el último (mes de mayo) se observó la evolución de la intervención, la cual ha producido cambios en todos los bloques de contenidos, destacando un avance significativo en el área de la concentración, especialmente en la conducta de realizar la tarea sin distraerse, la cual la realizó casi siempre (Figura 4). El área de la atención mejoró en general, aunque se observó un ligero retroceso referido a la atención que prestaba a la maestra mientras explicaba la tarea. Por otro lado, el área de la impulsividad se redujo significativamente, encontrado grandes mejoras en comportamientos como la disminución de pegar a sus compañeros sin ningún motivo y aumentaron sus relaciones sociales, además de comportamientos como mostrar afecto por sus compañeros/as y viceversa. Por último, en el contenido de autoestima, se registraron mejoras al sentirse más seguro consigo mismo.
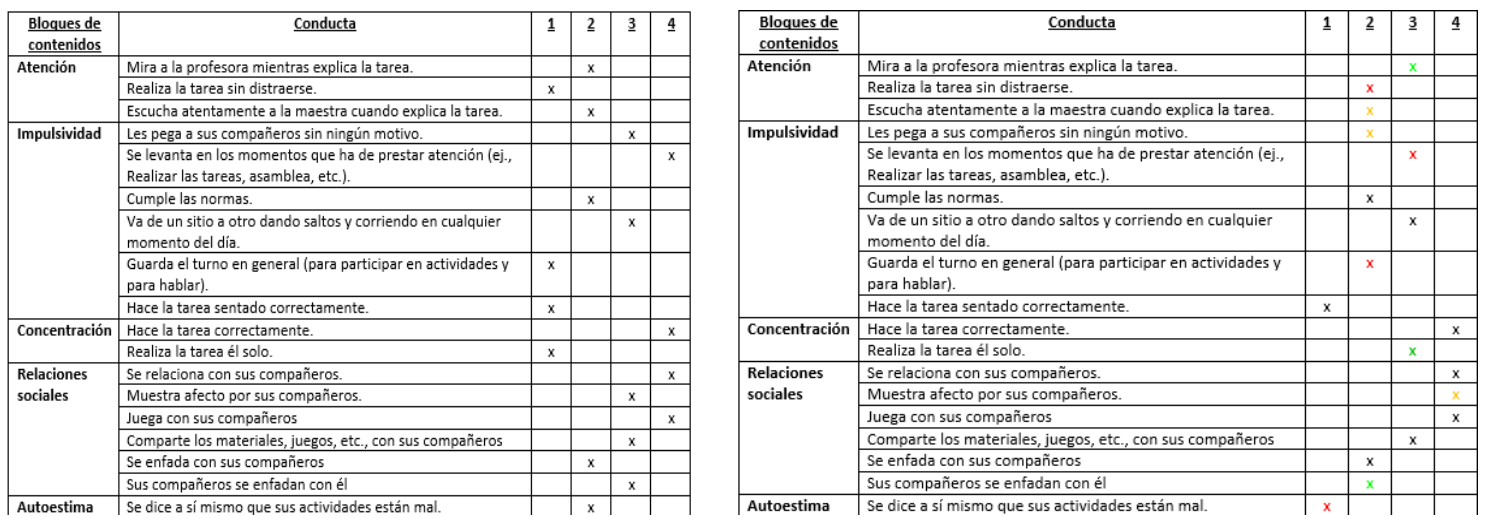

Figura 4: Comparación de registros observados meses Febrero vs Mayo 


\section{Estudio de casos 5}

Los resultados obtenidos después de la intervención destacan cambio, debido al gran progreso observado durante el primer mes de intervención. En este sentido, al comparar el primer -mes de febrero-, con el segundo registro -mes de marzo- se observó un aumento sobre el control de ambos idiomas, de la relación bidireccional con sus compañeros/as tanto en el aula como en el recreo, lo cual repercutió en el inicio para crear amistades con su grupo de mesa y a participar en las conversaciones con ellos.

Por otro lado, al realizar las comparaciones del segundo -mes de marzo- con el tercer registro -mes de abril- se registraron mayores progresos, especialmente en la categoría de la socialización, donde su integración en el aula fue un punto destacable. Durante este período la comunicación con sus compañeros/as aumentó, lo cual fortaleció aspectos como la confianza, logrando mantener sencillas conversaciones con otros alumnos/as, además, las frases que utilizó para comunicarse fueron en castellano. Asimismo, la relación con la tutora también ha aumentó, aunque se observó conductas de evitación en algunas situaciones.

Por otro lado, en las comparaciones entre el tercer -mes de abril- y el cuarto registro -mes de mayo- se observó un afianzamiento en las conductas que se intervinieron. Destaca la realización de frases para comunicarse en un único idioma -castellano- evitando así utilizar, como al inicio, la mezcla de los dos idiomas. También, la relación con los compañeros y otros alumnos del centro continuó aumentando, sobre todo en el tiempo de recreo. No obstante, la relación con la tutora se mantuvo de forma adecuada y mejorable.

Finalmente, al comparar el primer registro realizado con el último se observó una evolución totalmente favorable en cada bloque de contenidos. En la categoría de idioma todas las conductas mejoraron totalmente, y en la categoría de socialización, sólo la conducta relacionada con la interacción entre alumna y tutora, aunque con progresos elevados, no llegó a producirse la relación de confianza y apoyo deseada.

\begin{tabular}{|c|c|c|c|c|c|c|}
\hline CATEGORÍA & ÍTEMS & \multicolumn{5}{|c|}{ ESCALA } \\
\hline \multirow{5}{*}{ Idioma } & & 1 & 2 & 3 & 4 & 5 \\
\hline & $\begin{array}{l}\text { Confusión entre ambos idiomas. En una misma frase } \\
\text { encontramos palabras en ambas lenguas. }\end{array}$ & & & & & \\
\hline & $\begin{array}{l}\text { Se comunica en diferentes situaciones del ámbito escolar } \\
\text { (recreo, asamblea). }\end{array}$ & & & & & \\
\hline & Expresa frases sencillas en castellano. & & & & & \\
\hline & Se comunica con sus compañeros de mesa. & & & & & \\
\hline \multirow{4}{*}{ Socialización } & Se relaciona con sus compañeros. & & & & & \\
\hline & Los compañeros se relacionan con ella. & & & & & \\
\hline & Se relaciona con la tutora. & & & & & \\
\hline & Participa en actividades grupales. & & & & & \\
\hline
\end{tabular}

Figura 5: Comparación de registros observados meses Febrero vs Mayo 


\section{Discusión}

Los resultados hallados indican una mejora en la mayoría de los objetivos propuestos para cada caso concreto, así como el aumento en el desempeño y aprendizaje del alumnado de educación superior.

En el estudio de caso 1 la intervención, a nivel general los resultados obtenidos han sido favorables ya que el alumnado ha aumentado su puntuación en los diferentes ítems a lo largo del tiempo, no obstante, sólo se han conseguido los objetivos 1 y 3 , mientras que los objetivos 2 y 5 se han conseguido parcialmente y el objetivo 4 no se consiguió. Esta intervención ha tratado favorecer el desarrollo de aquellos niños en riesgo o que presentan dificultades ofreciéndoles soporte mediante una atención individualizada, al igual que los estudios de Caurcel Cara (2015), y sobretodo al realizar esta intervención en edades tempranas, se puede hablar de la plasticidad cerebral como la capacidad que tiene el cerebro para adaptarse a cambios y modificar el aprendizaje (Mulas Delgado y Hernández Muela, 2004), ha sido de gran importancia ya que se ha observado una adquisición de aprendizajes imprescindibles tanto para su desarrollo y adaptación en el medio natural, además, de mejorar sus capacidades de adaptarse a los cambios y modificaciones del proceso enseñanza-aprendizaje.

En este sentido, las investigaciones de García Alarcón (2009), indican que para mejorar el área cognitiva del alumnado con discapacidad intelectual, propone el uso de una metodología multisensorial basada en la percepción de estímulos táctiles, olfativos, gustativos, visuales y auditivos que, a su vez, origina un mayor desarrollo de habilidades cognitivas, la cual mejora las habilidades cognitivas, al igual que los resultados favorables extraídos en este caso, donde se utilizaron diferentes estímulos visuales, táctiles y auditivos.

Asimismo, los estudios de Ararú (2007) mencionan que la atención de los niños con discapacidad intelectual es breve $y$, por ello, se recomienda que las actuaciones no superen los 15 minutos ya que una mayor duración conlleva la perdida de la atención y aumenta el cansancio. En este sentido, las actuaciones realizadas se ajustaron a esta casuística y se consiguió mantener su atención el máximo tiempo posible, aumentando su concentración a la hora de realizar las tareas, lo cual ayudó en la adquisición de un aprendizaje progresivo, aunque si se registró un decrecimiento de esta conducta durante el último mes debido a que las actividades planificadas fueron extensas, y aumentó el cansancio acumulado de todo el curso escolar. Por otro lado, Valdivia (2002) indica que el diseño de actividades psicomotrices aumenta los efectos mejorando la coordinación motora, estructuración corporal y sentido rítmico, al igual que en este estudio, la intervención enfocada a este punto repercutió en mejoras del control postural, o movimientos óculos-manuales, aunque durante el último mes se observaron retrocesos en estos puntos, pudiendo ser ocasionados por una disminución en la motivación, ausencia a las sesiones, entre otras.

Si bien, se considera que la intervención realizada ha repercutido en mejoras, existieron una serie de limitaciones que pudieron afectar a los resultados finales, destacando:

- Conductas que no se pudieron trabajar por la falta de tiempo, impactando negativamente en un aumento del desarrollo madurativo del alumno.

- No poder trabajar exclusivamente con el sujeto del estudio.

- La ausencia regular a clase produjo que un gran número de sesiones no se implementaran. 
- Debido a que asiste al logopeda y al profesor de Pedagogía Terapéutica y debe de completar un material adaptado propio, no se dispuso del tiempo esperado, pudiendo sólo desarrollar las actuaciones dos sesiones a la semana.

- No poder intervenir con la familia del alumnado, tal y como Clotet i Serra y Virgill i Miró (1995), afirman que las intervenciones deben realizarse en un entorno cotidiano y habitual.

Aunque se observan progresos, sería recomendable introducir mejoras para futuras intervenciones que ayudarían a aumentar los resultados obtenidos en el proceso de enseñanza-aprendizaje, ampliar comportamientos deseables en el entorno escolar y familiar, además de unir fuerzas y líneas de trabajo entre ambos contextos, como por ejemplo, comenzar la intervención desde el inicio del curso escolar (septiembre), realizar sesiones de forma periódica y con cierta continuidad, aumento de la asistencia a las sesiones diseñadas previamente, o desarrollar actuaciones de trabajo específicas con la familia del alumnado para su implementación en el ámbito familiar.

Respecto al estudio de caso 2 se puede afirmar que se ha conseguido el objetivo 1 propuesto y el objetivo 2 parcialmente. Estos resultados se reflejan en otros estudios similares, donde la intervención temprana para prevenir las dificultades de aprendizajes juega un papel fundamental (Alfonso, et al., 2017; López y Guevara, 2008), así como repercutir en prevenir problemas o situaciones de riesgo, además de realzar un diagnóstico temprano en el caso de la existencia de algún trastorno.

Torres (2016) indica que los niños en el segundo ciclo de Educación Infantil se encuentran en la mitad de lo que Piaget (1983) llamó periodo preoperatorio, fase donde los niños ya son capaces de construir y utilizar símbolos mentales. No obstante, se ha de tener presente las diferencias personales y la maduración, las cuales les proporcionará los recursos para pasar por las diferentes etapas (desarrollo evolutivo), y en especial, se debe poner un mayor énfasis en la madurez del niño/a en el inicio de la lectura. En esta línea, este a lo largo de este estudio se ha observado que el sujeto no ha adquirido aún las capacidades y destrezas necesarias para iniciarse en la lectoescritura y, por lo tanto, deberían de desarrollar actuaciones concretas adaptadas, eficaces y efectivas. En esta misma dirección, el estudio realizado por Escotto (2014) en lectoescritura, tras la intervención, los participantes aún presentaban algunas dificultades, como por ejemplo lentitud, omisión de algunos fonemas o problemas para comprender textos, algo similar a esta intervención.

Por otra parte, los resultados de la investigación de González, Martín y Delgado (2010) demuestran, que los sujetos con riesgo de presentar dificultades de aprendizaje que fueron instruidos a través de su programa, mejoraron en lectura y escritura, mientras que los alumnos que no participaron, mejoraron a partir de los siete años. Esta conclusión, lleva a pensar que, con la intervención realizada, comprobadas las mejoras observadas pueden ser atribuidas a la ayuda introducida.

Sin embargo, este estudio presenta una serie de limitaciones, tales como las siguientes:

- Las actuaciones implementadas después del recreo influyeron negativamente en la consecución de los objetivos planteados, ya que la atención disminuía a lo largo del día, y repercutió en la concentración.

- Imposibilidad de modificar y adaptar la programación didáctica de la tutora a los cambios sugeridos para el desarrollo de puntuales actuaciones.

- Escasa duración del programa de intervención. 
- Falta de asistencia reiterada por parte del sujeto de estudio, lo cual supuso modificar la planificación establecida.

Es por ello, que para futuras experiencias surge la necesidad de introducir mejoras, tales como un incremento en la duración de la intervención (realizarse durante todo el curso escolar), o crear un horario estable y adaptado de actividades, las cuales se realicen en las primeras horas del día, ya que favorece la concentración en las actividades.

La intervención del estudio de caso 3, aunque se observan mejoras y progresos, los tres objetivos propuestos se han cumplido parcialmente. Tal y como indican Maganto y Cruz (2004), la etapa de infantil es el momento clave para detectan las dificultades, la cuales una vez son confirmadas por el médico, se realiza una observación detallada, y para este caso se propusieron medidas concretas (p.e., asignación de profesor de apoyo), lo cual está proporcionando beneficios a su desarrollo y se circunscribe a los objetivos y principios marcados para la atención temprana.

Asimismo, este trabajo coincide con Maganto y Cruz (2004), respecto al progreso en las dificultades observadas, las cuales pudiendo derivarse de aspectos como el esfuerzo realizado por el propio alumno y/o la ayuda recibida durante la intervención.

Por otro lado, Ruiz (2014) sugiere que un factor importante en la mejora de las dificultades es la motivación hacia todo tipo de actividades, siendo evidente en este caso su interés por aquellas actividades que fueron dinámicas, pudiendo ser un elemento que repercutiera en los resultados obtenidos. Otro componente con una carga significativa para la mejora de dificultades es el trabajo en habilidades sociales, las cuales repercuten en una mejor socialización con el grupo-clase o el respeto de normas, entre otras (Ruiz, 2014), y al igual que en este trabajo que introdujo medidas en esta línea los resultados fueron positivos tras la intervención.

No obstante, en este estudio se hallaron dificultades que pudieron incidir en la planificación de la intervención, e incluso en los resultados finales, destacando:

- Tiempo de trabajo real, el cual estuvo determinado, por un lado, a las reiteradas ausencias del alumnado, lo cual no permitió una rutina y consolidación de la intervención, perdiéndose a lo largo del tiempo el progreso alcanzado en algunas sesiones y, por otro lado, la duración de algunas sesiones (escaso tiempo de duración) que provocaron una reducción en el tiempo de trabajo y, por ende, en el avance del alumnado.

- Complicada atención individualizada, debido al número de alumnos/as en la misma aula, no se pudo ofrecer el tiempo necesario y así satisfacer eficazmente las necesidades del alumno de este estudio.

- Escasa participación e interés por parte del alumno marcando, en ocasiones, la imposibilidad de realizar actividades ya programadas.

- Influencia de comportamientos no adecuados establecidos desde el inicio de la intervención que influyó negativamente sobre la intervención.

En este sentido, se recomiendan una serie de mejoras a implementar en futuras investigaciones, tales como la de realizar un trabajo constante, es decir, iniciar al alumnado en rutinas diarias. También, sería imprescindible aumentar el tiempo de intervención (comenzar al inicio del curso escolar), lo cual repercutiría en una adecuada interiorización de los temas trabajados y mejoraría el desarrollo educativo. Además, de planificar actuaciones partiendo de sus intereses y preferencias, y distribución de actividades diseñadas. También conocer las diferentes actuaciones y/o 
propuestas realizadas por los profesores de apoyo, lo cual proporcionará información adicional con la que construir actuaciones más específicas. $\mathrm{Y}$, por último, sería conveniente contar con el apoyo de la familia para favorecer y afianzar el desarrollo del alumno.

En el estudio de caso 4 a nivel general los resultados obtenidos han sido favorables ya que el alumnado ha incrementado su puntuación en diferentes ítems durante la intervención, no obstante, sólo se han conseguido los objetivos 2 y 5 , mientras que los objetivos 1,3 y 4 se han conseguido parcialmente. Estos resultados están en la línea de otras investigaciones (Esteban, 2013; Prada, 2016; Villa, 2015), donde la propuesta de actividades relacionadas con la atención influye positiva y significativamente en este aspecto, tal y como se observa en este estudio.

Siguiendo el estudio de Esteban (2013) se perciben progresos en aspectos como en la impulsividad, problemas conductuales y relaciones sociales, las cuales tienen un efecto positivo en la motivación, y al igual que en este estudio, se han observado estos cambios, poniendo especial hincapié en la disminución de conductas entendidas como problemáticas, como esperar el turno de palabra y permanecer sentando en los momentos de trabajo, con lo que mejoró sus relaciones con el grupo clase, además de incrementar su autoestima.

Igualmente, el trabajo de Villa (2015) muestra cómo una intervención basada en los ámbitos psicológico, psicopedagógico y farmacológico, además de proporcionar ayuda a la familia, mediante el desarrollo de actividades para la resolución de problemas y utilizando programas de entrenamiento autoinstruccional mejora los síntomas nucleares del TDA/H. Pero si además se añade un enfoque educativo centrado en el alumnado, como se desarrolló esta intervención, también se registraron mejoras que repercutieron positivamente en el comportamiento con un aumento de sus capacidades generales.

Por su parte, Prada (2016) a través de una intervención individualizada y personalizada, demuestra el impacto y mejora en niños/as que presentan TDA/H, la cual está dirigida hacia actividades de autorregulación, relajación, resolución de conflictos, etc., consiguiendo mejorar comportamientos disruptivos, la capacidad cognitiva, o sus habilidades sociales. Al igual que este trabajo, su enfoque dirigido en actividades relacionadas con la relajación y resolución de conflictos principalmente, obteniendo mejoras en comportamientos disruptivos, aumento de las habilidades sociales y disminución de comportamientos impulsivos.

Por otro lado, durante la realización de esta intervención se detectaron diferencias entre la planificación inicial prevista y la implementación realizada. Estas diferencias se corresponden con una serie de dificultades halladas, destacando las siguientes:

- Insuficiente tiempo para la implementación de actividades.

- Ritmo de clase lento.

- La toma de decisiones sobre el alumnado era exclusiva de la tutora del grupo, por lo que limitaba la acción del alumnado en prácticas, dejando insuficiente tiempo para la propuesta de sesiones diferentes a las ya establecidas.

- La intervención no se pudo concretar en momentos fijos en el tiempo, sino que se desarrolló en función de las necesidades de la tutor/a del aula.

- La mayor parte del tiempo sólo se realizaban fichas del libro, siendo la principal prioridad.

- Imposibilidad de acceso al trabajo con las familias, ya que esta labor recaía exclusivamente en la tutora del grupo-clase. 
En esta línea, para poder mejorar estas circunstancias, en futuras réplicas se podrían introducir cambios que ayuden a mejorar la situación como, por ejemplo, aumentar el tiempo para el desarrollo de las actividades, el cual tiene una relación directa con el aumento del rendimiento. Además, introducir actuaciones dirigidas a las necesidades detectadas durante el tiempo que el alumnado de prácticas esté en el aula sin entorpecer el ritmo de la clase. Otro punto sería mejorar la organización y planificación del desarrollo de actuaciones concretas en el aula. También, el incremento del número de tutorías con los padres podría beneficiar al alumnado ya que conocer al sujeto en su ámbito familiar puede ayudar a diseñar actividades que le proporcionen herramientas de trabajo en el contexto escolar. Igualmente, por parte del centro, se podría mejorar el funcionamiento de actuaciones con respecto a las quejas de algunas familias sobre el trabajo extra a desarrollar en casa (cuadernillo de clase), ayudando a conocer lo realizado en clase y la necesidad de finalizar ciertas tareas en casa, mejorando así la relación entre el centro y familias, con la finalidad de crear un clima de colaboración y cooperación, que repercuta en un aumento rendimiento escolar.

La intervención del estudio de caso 5 cumple con los objetivos propuestos. Esta intervención ha tratado de mejor los aspectos de comunicación e integración de una alumna, donde la adquisición de vocabulario y estructura gramatical han influido en la capacidad de comunicación en su segunda lengua -castellano-, lo cual repercutió positivamente en el nivel social con el resto de sus compañeros/as. Además, siguiendo a Millán (2013) los niños bilingües tienen una mayor capacidad de su nivel lingüístico y control sobre su lenguaje ya que es más amplio que el de alumnos monolingües, lo cual puede fomentar un nivel mayor en su rendimiento escolar, tal y como se ha observado en este estudio. Por otro lado, factores sociales y psicológicos, según Ortega (2010), son factores que están relacionados con el éxito del aprendizaje de un nuevo idioma, como puede ser la perspectiva de la persona inmigrante hacia las personas que le rodean, o la motivación que tendrá para el aprendizaje del nuevo idioma y poder comunicarse de manera favorable. En este sentido, la elevada motivación por parte de la alumna en todo el proceso fue un factor relevante para su mejora, al igual que indica Maquilón y Hernández (2011), donde la motivación es el motor que mueve toda conducta, la cual permite obtener cambios en el alumnado. Por otro lado, Achotegui (2008) afirma que aproximadamente un tercio de las personas inmigrantes que viven en España sufren el llamado Síndrome de Ulises, el cual está relacionado con el estrés al que están sometidas las personas inmigrantes al ser rechazadas en su país de acogida, teniendo en cuenta este aspecto para la intervención, las actuaciones tuvieron en cuenta su bienestar, y lograr los objetivos propuestos, lo cual impacta en un incremento del autoconcepto, autoestima, confianza y bienestar psicológico, como sugieren otros estudios (Asher y Gazelle, 1999; Martínez, Inglés, Piqueras y Oblitas, 2009; Segrin y Taylor, 2007).

Pero, aunque se han observado factores que se ajustan a otros estudios, como por ejemplo los realizados por Ortega (2010) sobre la enseñanza de una nueva lengua en estudiantes inmigrantes y su integración en un nuevo país o por Millán (2013) centrado en la importancia del fomento del bilingüismo en edades tempranas, se han de tener en cuenta las limitaciones observadas durante la implementación del programa:

- Corto periodo de tiempo (desde febrero a mayo), lo cual impidió un mayor avance en el desarrollo de las competencias lingüísticas.

- Reiteradas faltas de asistencia, lo cual repercutió en la planificación previamente establecida, teniendo que reemplazar algunas sesiones.

- El uso de fonemas que no coinciden en ambas lenguas (inglés y castellano), en concreto la pronunciación del fonema / $\mathrm{r}$.

- Diferencias en la pronunciación y sonidos vocálicos en ambos idiomas. 
Partiendo de estos factores, en futuras intervenciones se deberían de tener en cuenta las siguientes mejoras que ayudarán a mejorar esta experiencia como, por ejemplo, el aumento del periodo de intervención (curso escolar completo), mayor número de sesiones de trabajo, las cual pudieran proporcionar un mayor número de herramientas y conocimientos. Otro aspecto, sería aumentar el número de participantes en la experiencia, con los que se hubieran podido trabajar aspectos como la socialización o integración de otras actividades, así se podría haber mejorado la eficacia y eficiencia de la intervención, además del desarrollo de actividades dirigidas al enfoque emocional.

Finalizado este estudio, se observa la importancia de trabajar en las necesidades individuales detectadas en relación a la adquisición de nuevos conocimientos, habilidades, destrezas y competencias por parte del alumnado, bajo un proceso de asesoramiento, acompañamiento y guía del mismo, mediante el diseño de acciones personalizadas, lo cual hace necesario partir de orientaciones y recursos concretos que ayuden en la instrucción de este trabajo y sitúen al alumnado en un marco amplio donde movilice el conocimiento para adaptarlo a nuevas situaciones. Tal y como estableció Esteve (2003), el alumnado necesita manipular el conocimiento, ponerlo al día, seleccionar lo que es apropiado para un contexto específico, aprender de manera permanente, entender lo que está aprendiendo, y que este le permita adaptarlo a situaciones que se transforman rápidamente, lo cual conlleva el afianzamiento de nuevas concepciones, enfoques y nuevas prácticas pedagógicas.

Esto queda reflejado en las conclusiones extraídas de los diferentes estudios:

- En el estudio de caso 1 se concluye que al trabajar en una edad temprana donde se diseñe una intervención ajustada y adecuada a las dificultades o necesidades será de gran relevancia, ya que se trata de la etapa más plasticidad, la cual facilita al cerebro la adaptación a los cambios necesarios, además de la adquisición de aprendizajes imprescindibles para un mejor desarrollo evolutivo.

- Respecto al estudio de caso 2 esta investigación supuso un gran avance y reto, logrando ampliar conocimientos teórico-prácticas relativos a la Educación Infantil. Mediante la implementación de las actividades propuestas, se observó el progreso constante (inicialmente no diferenciaba las vocales, pero finalizada la experiencia consiguió leer frases simples y cortas). Además, se pusieron en práctica actividades innovadoras y motivantes, frente a una metodología tradicional, siendo constatable la necesidad de reciclaje permanente para garantizar una educación de calidad.

- Mientras en el estudio de caso 3 fue satisfactorio conectar los conocimientos teóricos adquiridos durante los años de formación académica y, comprobar la utilidad de la intervención implementada, al lograrse las metas establecidas y el desarrollo de sus potencialidades, observado mediante los cambios de comportamiento y mejoras en las dificultades presentadas inicialmente, poniendo de relieve la importancia y los beneficios aportados desde la Atención Temprana a la etapa de Educación Infantil.

- En el estudio de caso 4 se puede afirmar que trabajando aspectos tales como la atención, impulsividad, relaciones sociales, o autoestima, entre otras, se puede mejorar el comportamiento y aspectos personales, que influyen directamente a un aumento del rendimiento escolar y profesional.

- En el estudio de caso 5 la intervención destaca el cambio a nivel lingüístico y social, principalmente debido a que las actuaciones se diseñaron de manera personalizada, lo cual favoreció la mejora de sus competencias básicas y le 
permita desarrollarse en su entorno de una manera bilingüe, lo cual le aportará habilidades y destrezas para el desempeño de su vida personal y laboral.

En definitiva, la aportación de este trabajo gira en torno a la idea de ajustar el nivel de desarrollo a las necesidades individuales del alumnado, con el que respetar la diversidad de las aulas y proceder con las estrategias educativas, recursos e iniciativas más adecuadas y efectivas que ayuden al progreso de los estudiantes mediante esfuerzo, conocimiento de aptitudes, actitudes, proceso de actuación ajustado a su necesidad, comunicación, profundización y ampliación de puntos de vista diferente, seguimiento y orientaciones ajustadas.

\section{Referencias}

Achotegui, J. (2008). El síndrome de Ulises no es comedia. El País-Salud, 10

Alfonso, S., Deaño, M., Ramos, A., Conde, A., Gayo, ME., Iglesias, V., Limia, S. y Tellado, F. (2017). Prevención e Intervención de las Dificultades de Aprendizaje en la Lectura. $3(1), 71-81$.

Ararú, A. (2007). Menores con Discapacidad y Necesidades Educativas Especiales. México: SEP.

Asher, S., \& Gazelle, H. (1999). Loneliness, peer relations, and language disorder in childhood. Topics in Language Disorders, 19(2), $16-33$.

Berrocal, E. (2016). Fundamentos e implicaciones de la observación en Educación Infantil. Observación sistemática y análisis de contextos en Educación Infantil. Granada: Universidad de Granada.

Bolívar, A. (2009). Aprender a aprender a lo largo de la vida. Multitarea. Revista de Didáctica, 4, 63-96.

Díaz, F. y Hernández, G. (2002). Estrategias docentes para un aprendizaje significativo. Una interpretación constructivista. México: McGraw-Hill Interamericana

Caurcel Cara, MJ. (2015). La atención temprana de los trastornos el desarrollo. En MD. López Justicia y MT. Polo Sánchez (coords.), Trastornos del desarrollo infantil (pp. 27-45). Madrid: Pirámide.

Clotet i Serra, N. y Virgill i Miró, M. (1995). La intervención logopédica en niños y niñas con plurideficiencias: una experiencia de trabajo multidisciplinar. Revista de Logopedia, Foniatría y Audiología, 25(4), 251-256.

Escoto, E. (2014). Intervención de la lectoescritura en una niña con dislexia. Pensamiento Psicológico. 12(1), 55-69.

Esteban, V. (2013). Tratamiento de un caso de Trastorno por Déficit de Atención e Hiperactividad. [Archived by WebCite $®$ at

http://www.psicoterapeutas.com/paginaspersonales/Violeta/td ah_caso.html]

Esteve, J.M. (2003). La tercera revolución educativa. La Educación en la Sociedad del Conocimiento. Barcelona, España: Paidós.
García Alarcón, GA. (2009). Impacto de estrategias didácticas multisensoriales para estimular el desarrollo de habilidades intelectuales de alumnos preescolares con discapacidad intelectual del centro de atención múltiple. $X$ Congreso Nacional de Investigación Educativa, 1, (pp. 1-11). Veracruz, México.

González, J. y Wagenaar, R. (2003). Tuning educational structures in Europe. I. Final Report. Bilbao, España: Universidad de Deusto.

González, M. J., Martín, I. y Delgado, M. (2010, 19 de febrero). Intervención temprana de lectoescritura en sujetos con dificultades de aprendizaje. Revista Latinoamericana de Psicología, 43(1), 35-44.

López, A., y Guevara, Y. (2008). Programa para prevención de problemas en la adquisición de la lectura y escritura. Revista Mexicana de Análisis de la Conducta, 34(1), 57-78.

Maganto, C. y Cruz, MS. (2004). Desarrollo físico y psicomotor en la etapa infantil. En MP. Bermúdez y AM. Bermúdez. (Coord.), Manual de psicología infantil: aspectos evolutivos e intervención psicopedagógica (pp. 27-64). Madrid, España: Biblioteca Nueva.

Maquilón, JJ., y Hernández, F. (2011). Influencia de la motivación en el rendimiento académico de los estudiantes de formación profesional. Revista Electrónica Interuniversitaria de Formación del Profesorado 14(1), 81-100.

Martínez, A., Inglés, C., Piqueras, J. y Oblitas, L. (2009). Papel de la conducta prosocial y de las relaciones sociales en el bienestar psíquico y físico del adolescente. Avances en Psicología Latinoamericana, 28(1), 74-84.

Martínez, J. (2011). Métodos de investigación cualitativa. Revista de investigación Silogismo, 1(8), 1-43.

Mateo, I. (2000). La evaluación educativa, su práctica y otras metáforas. Barcelona, España: ICE-Horsori.

Millán, S. (2013). Fomento del bilingüismo en edades tempranas: propuesta basada en el enfoque CLIL para el segundo ciclo de Educación Infantil (Trabajo Fin de Máster). Universidad de Oviedo, Asturias. 
Montero, I. y León, O. (2002). Clasificación y descripción de las metodologías de investigación en psicología. Revista Internacional de Psicología Clínica y de la Salud, 2(3), 503508.

Mulas Delgado, F., y Hernández Muela, S. (2004). Bases neurobiológicas de la atención temprana. En J. Pérez López y A. Brito de la Nuez (coords.), Manual de Atención Temprana (pp. 45-55). Madrid: Pirámide.

Ortega, LE. (2010). La enseñanza de la lengua en el proceso de integración del inmigrante (Trabajo Fin de Máster). Universidad de Jaén, Jaén.

Piaget, J. (1983). Seis estudios de psicología. Madrid: Ariel.

Prada, M. (2016). Estudio de caso único de un paciente de 12 años diagnosticado con TDAH presentación HiperactivaImpulsiva (Tesis de maestría). Universidad del Norte, Barranquilla-Colombia.

Ruiz, N. (2014). Entender y atender la diversidad: Estudio de caso en un aula de Educación Infantil (Tesis de grado). Universidad de Valladolid.

Rychen, DS. y Hersh, LH. (2002). Definición y selección de las competencias (DeSeCo): Fundamentos teóricos y conceptuales de las competencias. París, Francia: OCDE.
Segrin, C., \& Taylor, M. (2007). Positive interpersonal relationship mediante the association between social skills and psychological well-being. Personality and Individual Differences 43, 637-646.

Stake, RE. (2007). Investigación con estudio de casos. Madrid: Ediciones Morata.

Strauss, A. y Corvin, J. (2002). Bases de la investigación cualitativa. Técnicas y procedimientos para desarrollar la teoría fundamentada. Colombia: Editorial Universidad de Antioquia.

Torres, M. (2016). Lectoescritura: eventos de literacidad en preescolar. RIDE Revista Iberoamericana para la Investigación y el Desarrollo Educativo. 6(12), 1-17.

Valdivia, M. (2002). Actividades psicomotrices para el desarrollo de las coordinaciones motoras, estructuración del esquema corporal y sentido rítmico en niños especiales. Lima: Instituto Nacional de Estadística e Informática y Centro de Investigación y Desarrollo.

Villa, L. (2015). TDAH estudio de caso (Tesis de pregrado). Universitat Jaume I, Castellón.

Yin, RK. (1989). Case Study Research: Design and Methods, Applied social research Methods Series. Newbury Park CA: Sage. 
Anexos

\section{Anexo 1: Registro de observación Estudio de casos 1}

A continuación, se presentan las conductas a observar. Marcar con una " $X$ " el grado observado: $1=$ nada; $2=$ regular; 3 = bien; 4 = muy bien.

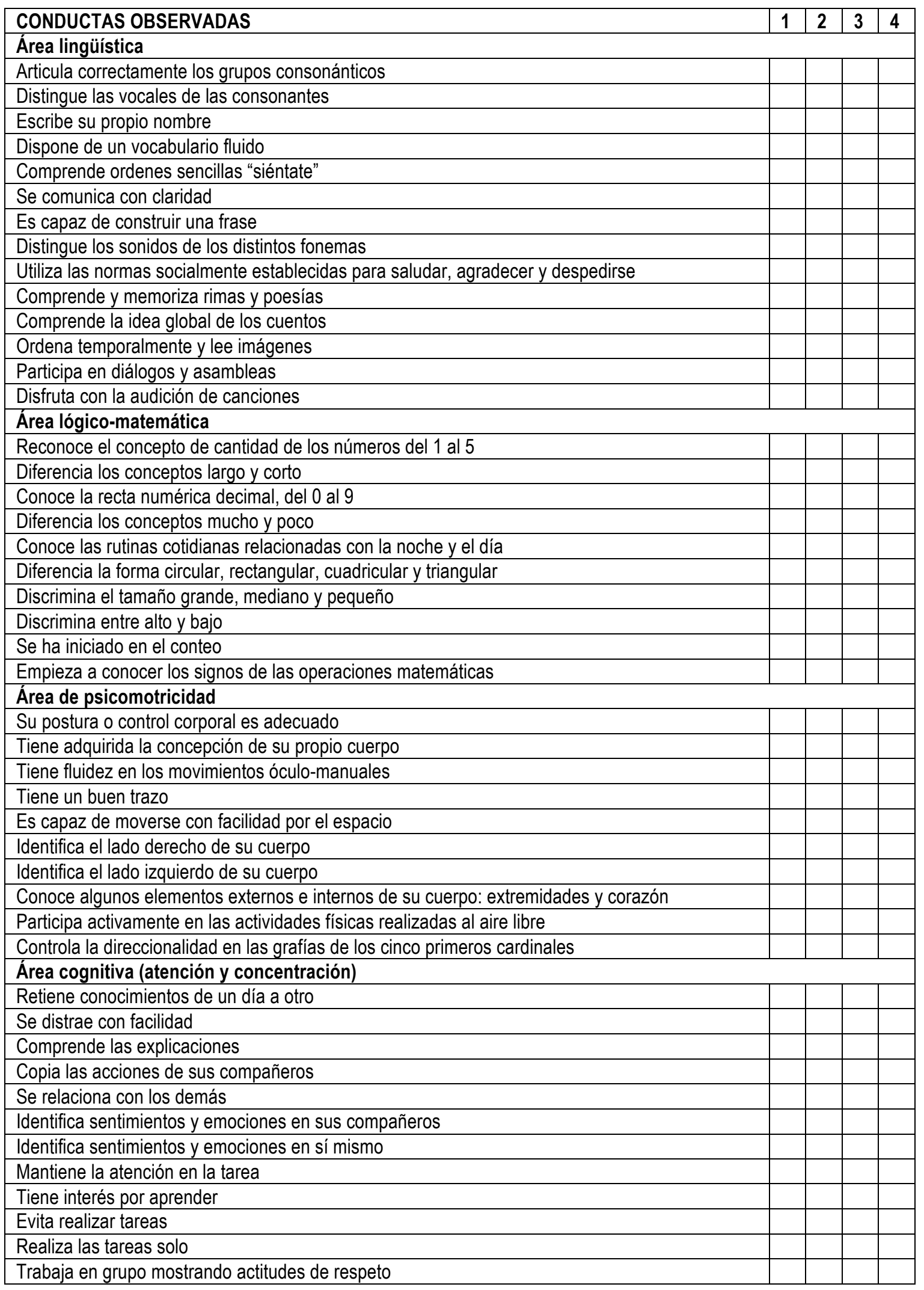




\section{Anexo 2: Registro de observación Estudio de casos 2}

A continuación, se presentan las conductas a observar. Marcar con una "X" el grado observado: $1=$ nada; 2 = a veces; 3 = a menudo; 4 = siempre.

\begin{tabular}{|c|c|c|c|c|}
\hline CONDUCTAS OBSERVADAS & 1 & 2 & 3 & 4 \\
\hline \multicolumn{5}{|l|}{ Lectura } \\
\hline \multicolumn{5}{|l|}{ Reconoce las vocales sin ayuda } \\
\hline \multicolumn{5}{|c|}{ Identifica las consonantes por sí sola cuando se le pregunta } \\
\hline \multicolumn{5}{|l|}{ Emite el sonido de las vocales } \\
\hline \multicolumn{5}{|l|}{ Pronuncia las consonantes de forma correcta } \\
\hline \multicolumn{5}{|l|}{ Dicen las sílabas que se le indican } \\
\hline \multicolumn{5}{|c|}{ Usa ayudas visuales para el reconocimiento de palabras } \\
\hline \multicolumn{5}{|c|}{ Sabe hacer los movimientos con la boca para pronunciar una letra } \\
\hline \multicolumn{5}{|c|}{ Relaciona la imagen que ve con la vocal por la que empieza } \\
\hline \multicolumn{5}{|c|}{ Enlaza la imagen con la palabra a la que se corresponde } \\
\hline \multicolumn{5}{|l|}{ Realiza las actividades sin ayuda } \\
\hline \multicolumn{5}{|l|}{ Le da un significado a lo que lee } \\
\hline \multicolumn{5}{|l|}{ Escritura } \\
\hline \multicolumn{5}{|l|}{ Coge el lápiz de manera adecuada } \\
\hline \multicolumn{5}{|l|}{ Realiza las vocales sin ayuda } \\
\hline \multicolumn{5}{|l|}{ Escribe las consonantes adecuadamente } \\
\hline \multicolumn{5}{|l|}{ Repasa los trazos correctamente } \\
\hline \multicolumn{5}{|l|}{ Escribe los números bien } \\
\hline \multicolumn{5}{|l|}{ Escribe su nombre } \\
\hline \multicolumn{5}{|l|}{ Sabe copiar la fecha de la pizarra } \\
\hline \multicolumn{5}{|l|}{ Copia la frase que se le indica en los deberes } \\
\hline \multicolumn{5}{|l|}{ Atención } \\
\hline \multicolumn{5}{|l|}{ Se distrae fácilmente } \\
\hline \multicolumn{5}{|l|}{ Juega con sus manos } \\
\hline \multicolumn{5}{|l|}{ Habla con otros compañeros } \\
\hline \multicolumn{5}{|l|}{ Saca la lengua cuando está pensando } \\
\hline \multicolumn{5}{|l|}{ Se mueve en la silla } \\
\hline Pide volver a hacer la actividad & & & & \\
\hline Quiere terminar la actividad & & & & \\
\hline Pone atención en lo que hace & & & & \\
\hline Se entretiene con los dibujos de las actividades & & & & \\
\hline Comportamiento & & & & \\
\hline Se tira al suelo & & & & \\
\hline Molesta a los compañeros & & & & \\
\hline Guarda silencio & & & & \\
\hline Se comporta de forma adecuada en la fila & & & & \\
\hline Se sienta bien en la Asamblea & & & & \\
\hline Respeta los turnos de palabra & & & & \\
\hline Escucha atentamente a los demás compañeros & & & & \\
\hline
\end{tabular}




\section{Anexo 3: Registro de observación Estudio de casos 3}

A continuación, se presentan las conductas a observar. Marcar con una "X" el grado observado: $1=$ nunca; $2=$ frecuentemente; 3 = a veces; $4=$ siempre.

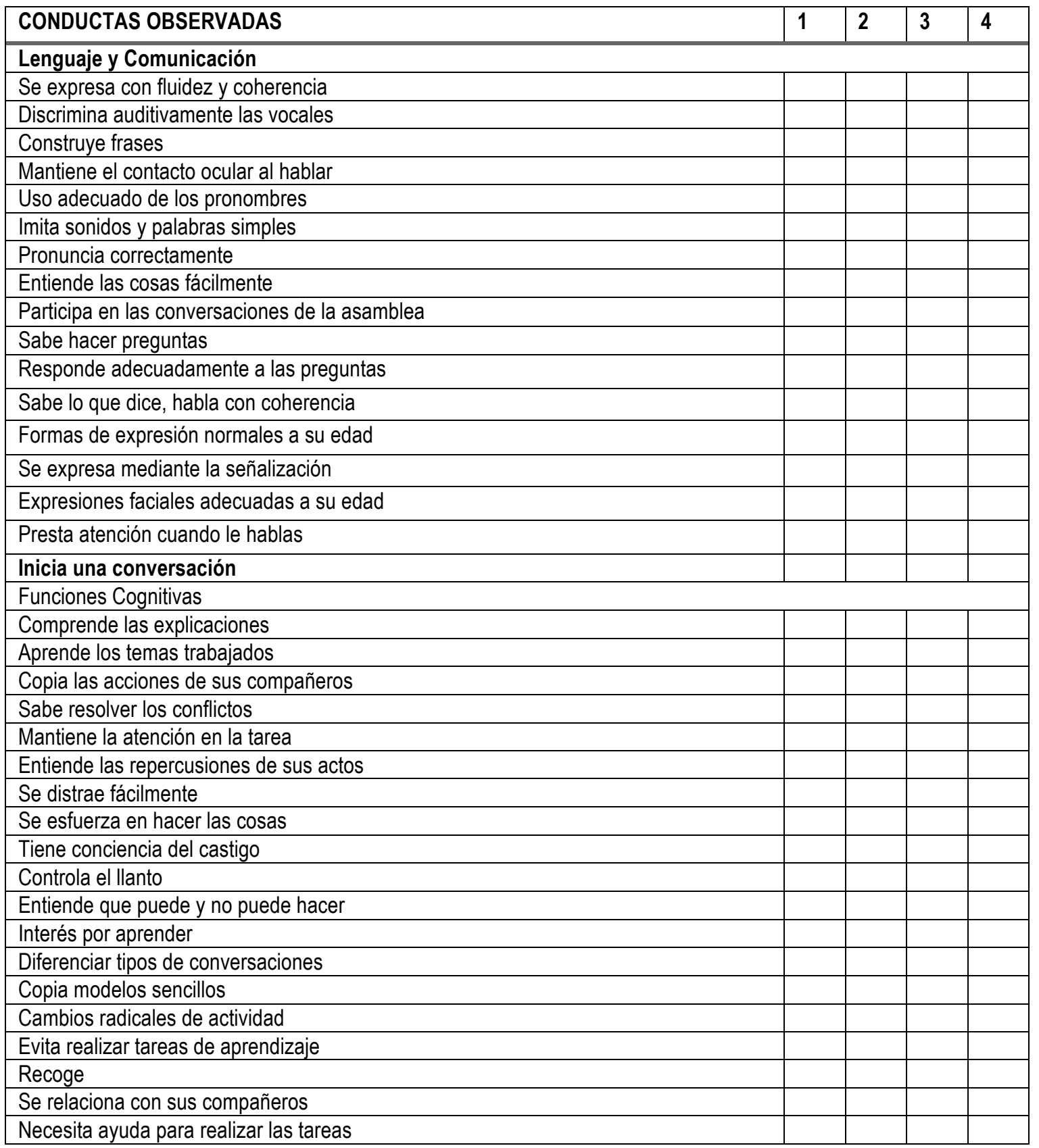




\section{Anexo 4: Registro de observación Estudio de casos 4}

A continuación, se presentan las conductas a observar. Marcar con una "X" el grado observado: 1 = nunca; 2 = a veces; 3 = casi siempre $4=$ siempre.

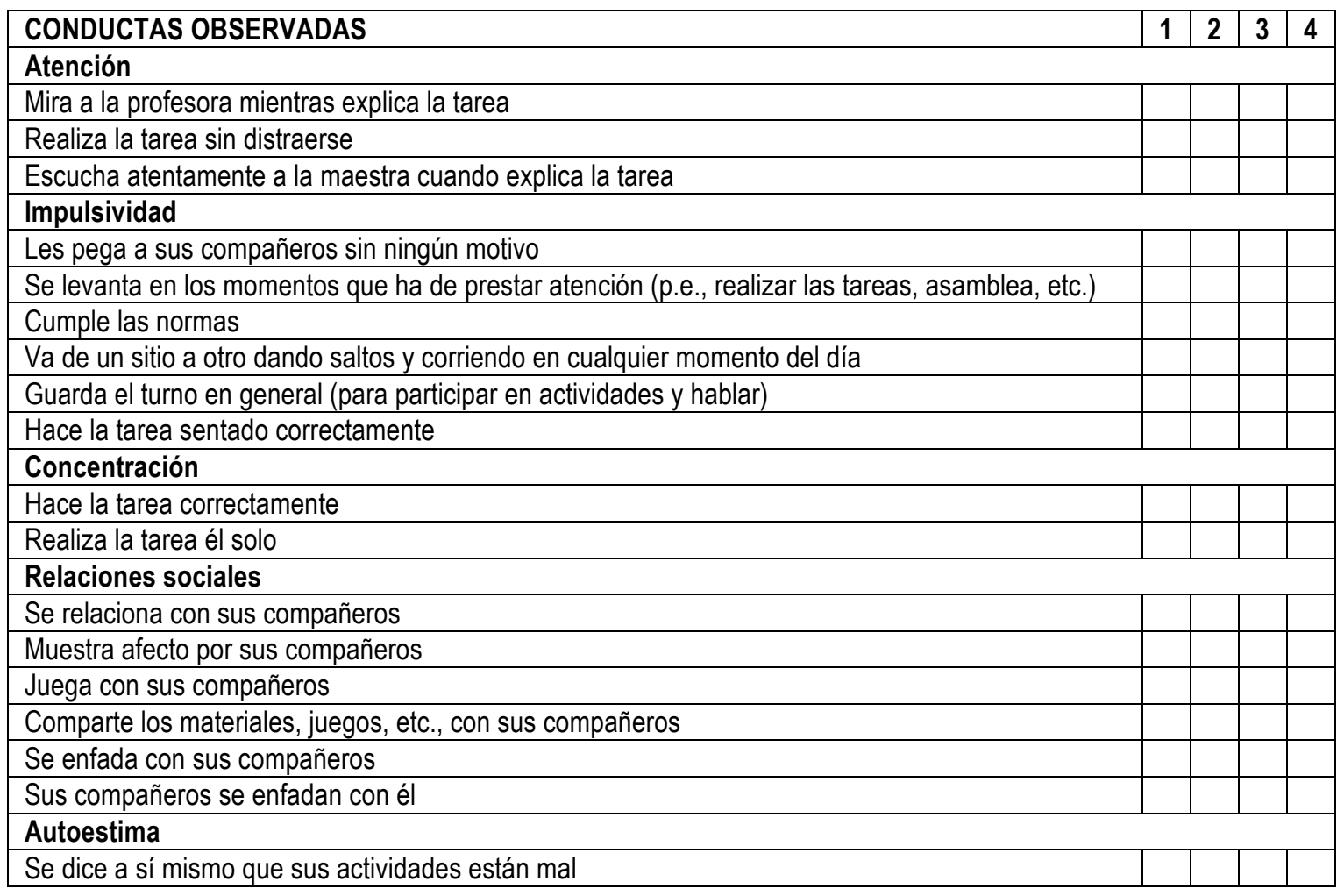

\section{Anexo 5: Registro de observación Estudio de casos 5}

A continuación, se presentan las conductas a observar. Marcar con una "X" el grado observado: 1 = nunca; 2 = pocas veces; 3 = con frecuencia; 4 = casi siempre; 5 = siempre.

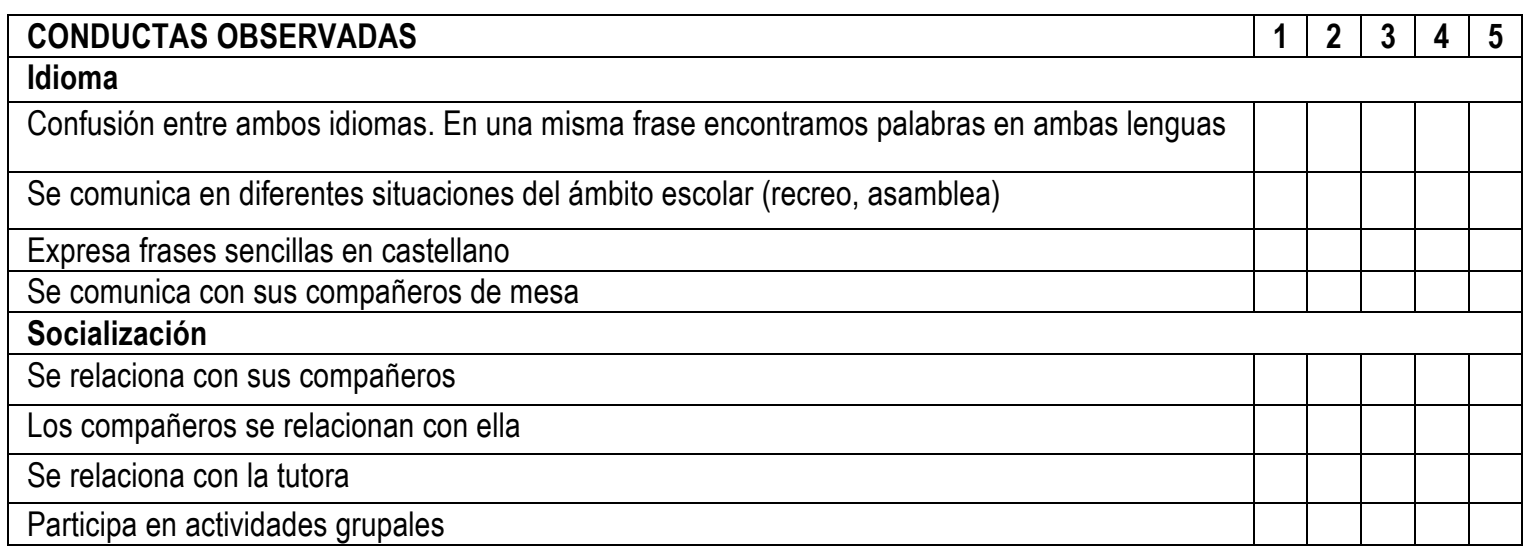

